Document downloaded from:

http://hdl.handle.net/10251/124282

This paper must be cited as:

Li Vigni, M.; Prats-Montalbán, JM.; Ferrer, A.; Cocchi, M. (2018). Coupling 2D-wavelet decomposition and multivariate image analysis (2D WT-MIA). Journal of Chemometrics. 32(1):1-20. https://doi.org/10.1002/cem.2970

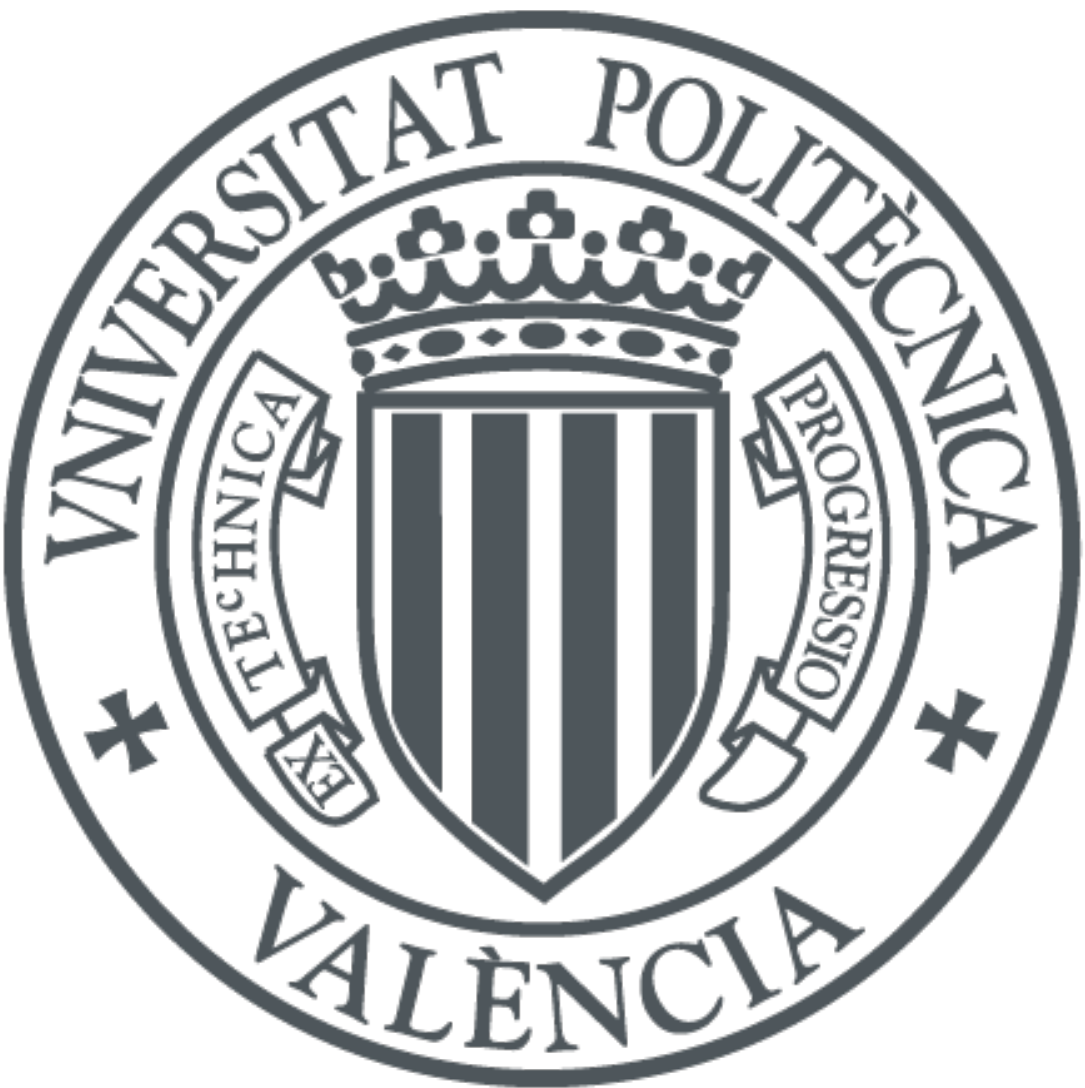

The final publication is available at

http://doi.org/10.1002/cem.2970

Copyright John Wiley \& Sons

Additional Information 


\section{Coupling 2D-Wavelet decomposition and Multivariate Image Analysis (2D WT-}

2

3

4

5

6

8
MIA)



\section{ABSTRACT}

The use of 2D Discrete Wavelet Transform in the Feature Enhancement phase of the Multivariate Image Analysis is discussed and implemented in a comparative way with respect to what already present in the literature. In the proposed approach, all of the resulting sub-images obtained by the Discrete Wavelet Transform decomposition are unfolded pixel-wise and mid-level datafused to a Feature Matrix which is used for the Feature Analysis phase. Congruent sub-images can be obtained either by reconstruction of each decomposition block to the original pixel dimensions, or by using the Stationary Wavelet Transform decomposition scheme. The main advantage is that all possible relationships among blocks, decomposition levels and channels are assessed in a single multivariate analysis step (Feature Analysis). This is particularly useful, in a monitoring context, when the aim is building multivariate control charts based on images. Moreover, the approach can be versatile to handle context where several images are analysed at a time as well as in the multispectral images analysis. 
25 Both a set of simple artificial images will be used to highlight the details of the

26 methodology and show how the wavelet transform allow extracting features that are

27 informative of how strong, and in which direction, the texture of the image varies and a

28 set of real images as representative of the on-line quality monitoring context.

29

30 Keywords: 2D Wavelet Transform, Multivariate Image Analysis, Multi resolution,

31 Quality monitoring 


\section{INTRODUCTION}

33 The use of Multivariate Analysis to evaluate images dates back to the mid-late 80 's,

34 with the work of Esbensen and Geladi [1] who introduced the use of Principal

35 Component Analysis for the study of multi-channel images. Multivariate Image

36 Analysis (MIA) has soon gained boost with the application in many contexts, typically

37 those with images of such complexity that they could benefit of a multivariate analysis

38 approach (e.g. remote sensing ${ }^{2-4}$ and medical imaging ${ }^{5-9}$ ). In the 90 's, the pioneering

39 works of MacGregor and his group made the field of process industry accessible by the

40 MIA approach ${ }^{2}$, 10 : the possibility to acquire images for on-line process monitoring purposes and effectively analyse them represents a viable, PAT-like sensor to investigate

42 process changes in time in an environment, that is the process line, where often the room for installing and interface new "traditional" sensors is poor, not to mention the

44 fact that often a single image can acquire simultaneously several different potential sources of variability.

46 At present, several uses of MIA are reported in literature for different tasks ${ }^{11-29}$, all of

47 which are characterised by being well described by the information an image can carry,

48 i.e. textural variability (based on the "two" dimension relationship structure of pixels)

49 and optical variation properties (based on the "third" dimension, that is the channels

50 acquired for each pixel). The latter aspect becomes the more relevant as the number of 51 channels increases, moving from simple binary or gray-scale images (where not much

52 information can be given more than texture homogeneity/non homogeneity), to RGB

53 images (where changes in colour can be related to the presence of non homogeneous

54 texture or underlying phenomena which alter the composition), to multi-channel images

55 and spectral images (where chemical information can effectively be acquired for each 
pixel). Therefore, most image-based challenges which can be addressed with MIA approach represent the detection of product defects in quality control $^{11-18}$, the monitoring of changes in process behaviour and its feed-back control ${ }^{16,}{ }^{19-21}$, the prediction of product properties on the basis of the joint evaluation of texture and channel information (this latter aspect is in particular addressed to by the development of Multivariate Image Regression - MIR - methods ${ }^{22-23}$ ); or more recently the development of imaging biomarkers in cancer diagnosis ${ }^{8-9}$.

As far as the core details of the MIA approach and its evolution in time, the MIA approach proposed by Bharati and MacGregor is based on a framework ${ }^{11-12}$ which can be summarised in two main steps: a Feature Extraction (or Enhancement) phase, in which the image (pre-processed, if necessary) is treated so that the texture information carried out by the pixels is made clearer, and a Feature Reduction (or Analysis) phase, where a suitable Multivariate Analysis method is applied (e.g. Principal Component Analysis, Partial Least Squares Regression, Partial Least Squares Discriminant Analysis) on the feature matrix obtained after the first phase. The two phases are strictly connected to each other, since the first step can strongly influence the outcome of the following analysis in a way, which is not much different from the effect of data preprocessing in many other situations. However, a certain degree of freedom can be considered when choosing the feature enhancement method (whilst the feature analysis phase is more application driven): the fundamental aspect to be preserved in this case is that it is not only important to preserve the information given by the channels, for which a simple unfolding of the image structure so that each pixel becomes a sample could be sufficient, but to retain the correlation among neighbouring pixels (that is, the texture information) and, most of all, present it to the following analysis step in such a way that 
80 both sources of variability (texture and channel-based properties) can be easily evaluated. In the approach proposed by Bharati and MacGregor ${ }^{2}$ the texture information is extracted by augmenting column-wise the unfolded pixel vector with a series of its copies, shifted row-wise so that each row of the generated matrix is formed by a pixel and all its surrounding neighbours. This corresponds to stacking copies of the original image shifted according to a given pace. The number of neighbours, hence of columns, of the feature matrix is $(2 w+1)^{2}$, governed by the window aperture parameter $w$, which indicates the dimension of the window, centred on the pixel, encompassing the neighbours to be considered (typically, $w=1$ or 2 ). In Prats-Montalbán et al. ${ }^{17}$ this augmentation is extended to each channel of the image, we will refer to this now on as colour-textural MIA (ct-MIA), while Facco et al. ${ }^{14}$ proposed a method to reduce the computation cost when operating with a larger window size, $w>2$. Other approaches have been proposed and discussed, among which the most common are based on the application of a transformation of the image, again for each channel, in a different domain, such as the Fourier domain (via the 2D Fourier Transform) or the wavelet domain $^{10-12,18,24-29}$. The wavelet advantage with respect to Fourier is that it has both good frequency and spatial resolution.

There may be several advantages by moving to wavelet domain in terms of image compression, background removal and denoising. Moreover, the use of wavelet transform allows a better understanding of the pixel correlation structure at different scales, since at each level of decomposition, the coefficients carry both the information pertaining to the energy which characterise a frequency range (based on the selected filter) and an indication about the orientation in which varies (according to the type of decomposition block, being it Approximation or one of the three Details blocks, namely 

transform can be seen as a truly enhanced visualization of how strong, and in which direction, the texture of the image varies. Literature differs in the way these features could be expressed and handled: some authors have pointed the attention to the use of global indicators to synthesise the relevant information for a given decomposition level and block, by using, e.g. the Frobenhius norm (Energy), the entropy, statistical momenta or the standard deviation of the coefficients ${ }^{18,26-28}$, thus having a single variable which summarises the effect, while maintaining the orientation information by means of the level-block combination at which it is computed. This approach surely reduces the computational cost of the following analysis, but carries with itself the potential loss of interesting information, which goes together with an "averaging" procedure of a richer set of data. Also, since all the information of an image is compressed in a single vector of descriptors for each decomposition block and level, a somehow conspicuous set of images has to be considered to create a reference set, for example of Normal Operating Conditions (NOC), when moving to the following Feature Analysis phase. On the contrary, working at pixel level, that is considering each pixel as a sample, opens the possibility to reduce the requirements when building a reference set, often allowing the use of a single representative image only - being it a real one, or a combination of snapshots of NOC texture areas.

123 Liu and MacGregor ${ }^{10}$ have proposed an approach where the wavelet transform is used for Feature Enhancement of images working at pixel level, i.e. the MultiResolutional Multivariate Image Analysis (MR-MIA). MR-MIA is conjugated in two frameworks that differ in the stage at which the wavelet transform is applied, i.e. before (MR-MIA I)

127 or after (MR-MIA II) the Feature Analysis step (in this case PCA). In particular, in MR- 
128 MIA I by applying the discrete wavelet transform (2D DWT) to each channel ch of the 129 image, each decomposition block, at a given level, can be seen as an image itself with 130 the same number of channels, but representing a different "resolution" and texture 131 orientation. The Feature Analysis (e.g. PCA) step is then applied to each of these 132 images, once unfolded pixel-wise, so as many latent variable models as number of 133 blocks per decomposition level (L) are obtained. This approach relies on the 134 orthogonality of the wavelet decomposition blocks, thus implying that there is no 135 interest in evaluating correlations among blocks at different scales, and the possibility to 136 evaluate texture - channel correlations is maintained. However, the complexity aspect 137 of this approach can be a hindrance when considering how many multivariate models one should compute and the necessity of a high-level data fusion step where all the results are combined to create a decision rule in order to e.g. decide if a new product image has to be rejected when compared to the NOC modelled one. Recently, basing on

141 similar considerations, Juneau et $a .^{25}$ proposed an approach where all sub images 142 obtained by wavelet decomposition, once unfolded pixel-wise, are merged row-wise and analysed by a single PCA. However, they used the continuous wavelet transform 144 (undecimated scheme, UWT) and in this way a rather large number of features is obtained, since scale and shift parameters vary continuously.

146 The MSMIA approach proposed by $\operatorname{Reis}^{18}$ is similar to the MR-MIA I, although more 147 images are considered at the same time as references NOC, but it differs in the way 148 information from the Feature Analysis step (e.g. PCA) of each WT decomposition block 149 is fused. In this approach, an index evaluating the distance to the scores distribution 150 histogram of the reference NOC images for each decomposition block, at a given scale, 151 is calculated in order to obtain a set of variables, which are then used for building a 
monitoring chart. In addition to this, multivariate control charts based on PCA of wavelet features (e.g. standard deviation of wavelet coefficients for each decomposition block), extracted for each decomposition block, at a given scale, are also considered. The approach is effective in compressing information and for on-line implementation, however defects location requires a further step. This step, similarly to MR-MIA II, consists in building a spatial shifting feature matrix (then analysed by PCA) for each of the sub-images contributing to "out of control" observations in the preceding step. Moreover, correlation structure among textural/colour pattern at different scales is only indirectly taken into account (the information from different scales is always merged at features, not pixels level).

Here we present an approach, which is named 2D WT-MIA, where the Feature Enhancement step, is similar to MR-MIA I, but as in Juneau ${ }^{25}$ all of the resulting subimages obtained by the 2D-DWT decomposition are unfolded pixel-wise and mid-level datafused to a Feature Matrix which is used for the Feature Analysis phase. In order to have congruent sub-images all decomposition blocks are reconstructed separately to the original pixel dimensions. This reconstruction step can be omitted, when the Stationary Wavelet Transform (2D-SWT) is used ${ }^{30-31}$. In this way, all possible relationships among blocks, decomposition levels and channels are assessed in a single multivariate analysis step (Feature Analysis). This is particularly useful, in a monitoring context, when the aim is building multivariate control charts based on NOC images. Thus, our proposed approach can be versatile to handle context where several images are analysed at a time as well as in the multispectral images analysis.

The rest of this paper is organised as follows: in Section 2: Methods, the proposed 2D WT-MIA approach is described into more details and compared to colour-textural MIA 
177 images which will be used will be presented, consisting in a set of simple artificial

approach to highlight common and differing aspects. In Section 3: Materials, the dataset binary images, used to illustrate how texture is captured within the two approaches and a set of real images, and in Section 4: Results and Discussion, these images will be analysed according to the two-step MR-MIA framework, using Principal Component Analysis as Feature Analysis technique with the target of simulating a quality control task.

\section{METHODS}

The approach here described belongs to the more general framework of MultiResolution Multivariate Image Analysis, thus basing on a two-phase elaboration of the image: a first step of Feature Extraction (Enhancement) and a second step of Feature Reduction (Analysis) (Figure 1). In particular, the 2D WT-MIA (wavelet based feature enhancement) and the colour-textural MIA (spatial shifting based feature enhancement) approaches will be discussed and compared in terms of results in the present work.

\subsection{Spatial Shifting Feature Enhancement}

Colour-textural MIA $^{17}$ is summarised in Figure 2. This approach to Feature Enhancement consists in capturing, for each channel $c h$, the pixel proximity correlation by means of a spatial shifting of neighbouring pixels with respect to each pixel of the original image, according to a selected window aperture parameter, $w$. In practice, starting from a pixel element of the image $p_{i, j}$, a row vector is created by adding the intensity value of the channel corresponding to the closest surrounding pixels: if $w=1$, the composition appears as reported in Figure 2. When this is done for all the pixels of a pixel-wise unfolded channel matrix, and the feature matrices for the different channels 
are then fused, a total Feature Matrix is obtained which has as many rows as the number of pixels $I=n_{1} \times n_{2}$, and as many columns as the number of channels $c h$ times the number of spatial shifted pixels, which is given by $(2 w+1)^{2}$. This means that, regardless of the number of channels, for a window parameter $w=1$ (the closest neighbours) the Feature matrix is $9 \times c h$, and when moving to $w=2$ (the closest neighbours and the next surrounding layer), the Feature matrix is $25 \times c h$. This implies a fast increase of the number of variables considered in the analysis, the higher is the number of channels.

Since we need, at each pixel location, to use all the neighbouring pixels until a distance $w$, this implies that we lack of information for all those pixels in the borders with width $w$. Theferore, the solution commonly adopted is to diminish the size of the image from $n_{1} \times n_{2}$ to $\left(n_{1}-2 w\right) \times\left(n_{2}-2 w\right)$

\subsection{D Wavelet-based Feature Enhancement}

Figure 3 shows the general scheme of the Feature Enhancement step involving 2D DWT application, through the fast Mallat algorithm ${ }^{32-33}$, on an image. For each channel ch, the low-pass and high-pass filters (which are the same as in the 1D case) are first operated row-wise on the image and then, after downsampling of the coefficients, in each of the resulting blocks column-wise. In this way four decomposition blocks are obtained: Approximations (low-pass+low-pass), namely CA; Horizontal details (low+high), namely $\mathrm{CH}$; Vertical details (high+low), namely $\mathrm{CV}$, and Diagonal details (high+high), namely $\mathrm{CD}$. The procedure is then iterated by applying it to the Approximations, i.e. increasing the decomposition level. Downsampling is skipped when the 2D - SWT scheme is used since, instead, the filters are up-sampled ${ }^{26}$. The 
maximum possible decomposition level, L, depends on the image size. The four decomposition blocks obtained from each level of decomposition (CA, $\mathrm{CH}, \mathrm{CV}$ and - DWT so that their dimensions are the same of the original image, while they are already of the same size when 2D SWT is used (in fact, each block of coefficients at every level maintains the same size as the original image, and congruent images are obtained). This leads to a set of $4 \times L$ images for each channel $c h$, which can be unfolded and column-wise merged to obtain a total Feature Matrix which has as many rows as the number of pixels $I=n_{1} \times n_{2}$, and as many columns as $4 \times L \times c h$. If we compare this column dimension to the one obtained with the Spatial Shifting approach, which is $(2 w+1)^{2} \times c h$, it might appear that there is little benefit in terms of reduction of the Feature Matrix dimensions. However, two aspects have to be underlined:

i) in the spatial shifting approach the image is analysed by moving a $(2 w+1) x(2 w+1)$ pixels window by step of 1 in all image directions; on the other hand, with wavelet, a filter length $x$ filter length pixels window is moved by step of 1 in all image directions, but using a larger filter does not increase the number of features, which remain always four;

ii) the two approaches lead to the same number of feature descriptors if $L=\operatorname{round}[(w+$

$2401 / 2)^{2}$ ]. This corresponds, for e.g. a window parameter of $w=2$, to a decomposition level $L=6$, which in terms of multiresolution means to have gone very deep in the analysis of coarse and smooth aspects of the image. In other words, such a decomposition level (if allowed by the nature of the chosen wavelet) usually leads to the possibility of

244 evaluating correlations and high distance relationships among pixels to an extent, which is superior to the use of a moving window of fixed size. 
246 When applying the wavelet transform, the selection of the most appropriate wavelet

247 filter is considered a critical issue and a limiting step in implementation of routine

248 applications (i.e. which wavelet family and which filter length, to analyse the specific

249 characteristics of the images at hand). This issue has been dealt in literature by 250 analysing the different properties of the decomposition filter in terms of texture 251 description capability in order to propose general criteria ${ }^{34}$ or focusing on goodness of 252 image reconstruction ${ }^{33}$, or proposing a design of experiments approach ${ }^{36}$. We recently 253 proposed ${ }^{37}$ a methodology based on $\mathrm{N}$-way modelling to provide a range of possible 254 wavelet choices (in terms of families, filters, and decomposition levels), for each image 255 and problem at hand. Any of these strategies requires a preliminary analysis step to be conducted by experienced people in the field, although this step is only required once in model building. However, some considerations, based on our experience can be drawn: i) there is in general a relation between the decomposition level and the filter length, i.e. by using a larger filter a lower decomposition level is required to capture the different image aspects (coarse and smooth) and ii) taking into account the wavelet families characteristics, such as for degree of symmetry or regularity, number of vanishing moments $^{38-39}$ it is possible to focus on a small number of wavelet filters to test, by choosing a representative one for each type of property.

\section{MATERIALS}

\subsection{Artificial Images datasets}

267 These sets are used to illustrate how the colour-textural MIA and 2D WT-MIA approaches analyse texture and their capability to detect faulty pixels. These images are 
269 characterized by two main features: a particularly limited pixel size, so that 270 computational time is not a relevant benchmark property at this stage, and a simple, yet 271 well defined, pattern. Also, the differences between "normal", i.e. reference image, and 272 "defective", i.e. image (or images) for which a perturbation of the pattern was created, 273 are well controlled, in the sense that the number and position of pixels which have been 274 changed is known, and the entity of the disturb is enough to obtain simulated test 275 images which are not too similar to their reference image. In spite of the simplicity of 276 this simulated case, the information which can be acquired from the analysis with both 277 approaches is interesting to better understand how the two methods under comparison 278 work, and the conclusions which can be drawn are helpful and can be extended, as 279 shown in the next section where real images are presented and dealt with, to cases of higher complexity.

The set is composed by three binary images, as reported in Figure 4, of pixel size $32 \times$ 32. Figure 4 "SimSetA" reports the "normal" (reference) image, on the basis of which an alternation pattern has been generated. In this case, the squares which alternate in both image directions to give a chequered pattern have a dimension of $8 \times 8$ pixels: starting from upper left corner and moving over columns dimension, a white (1's) $8 \times 8$ pixel square is alternated to a black ( 0 's) $8 \times 8$ pixel square, and the same alternated

287 pattern is repeated over the rows dimension. Starting from this image, two changes in pattern were produced, leading to two "defective" (test) images. Figure 4 "SimSetB" shows an overlying irregular shape which extends from the diagonal to the lower left part of the image: for this figure, a total of 55 pixels have been inverted in value (from 1 to 0 or from 0 to 1 ) over the total of $32 \times 32=1024$ pixels. Figure 4 "SimSetC" shows another change in the pattern, this time according to a regular shape which is applied on 
293

294

295

296

297

298

299

300

301

302

303

304

305

306

307

308

309

310

311

312

313

314

315

top of each of the $8 \times 8$ pixel squares: for each of these squares, starting from the second diagonal element, a single pixel every fourth has been modified both in the rows and in the columns, thus resulting in a change of four pixels for each of the squares. For this figure, a total of 64 pixels have been inverted in value (from 1 to 0 or from 0 to 1 ) over the total of $32 \times 32=1024$ pixels.

\subsection{Real Images datasets}

To further explore the performance of the method proposed in this work and compare it to the results of the ct-MIA approach, additional datasets have been taken into account, belonging to different applicative contexts, tiles and bread production, respectively. In both cases, the control of the final product undergoes visual inspection, while the datasets differ as for image dimensions and number of channels.

\subsubsection{Tiles}

These datasets come from a production of tiles of marble-like materials for surface coverage: all the cases share a common issue, that is presenting product samples which do not comply to a strict definition of "normal" images, characterized, for instance, by a precise colour shade or by the absence of defects such as spots and scratches. Therefore, it is necessary to develop a method, complementary or alternative to visual inspection, which is able to: a) recognize the presence of a defectiveness when a new tile is compared to the reference one(s); b) indicate the kind of defectiveness (e.g. colour shade and/or presence of unwanted changes in surface pattern); c) locate on the surface the position of the defect in order to obtain an enhanced perception of the same, so that its visualization and recognition by the operator is made easier. Samples from two different products were considered with different degree of irregularity of the pattern in 
316 the defective tiles. They both consists of RGB images of dimensions are $256 \times 256$

317 pixels (Figure 5). Figure 5a reports dataset 1: Blanco Zeus, from now on referred to as

318 BZdataset, which is composed by three reference images (BZN01, BZN02 and BZN03),

319 and three images of tiles showing defects (BZD01, BZD02, and BZD03). This kind of

320 tile shows a mostly homogeneous shade of gray all over its surface, so that defects (as

321 for instance white or dark spots and scratches) do not usually present particularly high

322 difficulty of detection also by visual inspection. Figure $5 \mathrm{~b}$ reports dataset 2: Blanco

323 Norte, from now on referred to as BNdataset, which is composed by three reference

324 images (BNN01, BNN02 and BNN03), and three images of tiles showing defects

325 (BND01, BND02, and BND03). In this case, the tile main colour is gray, but the surface

326 is characterized by an inhomogeneous distribution of darker spots, in a grainy structure,

327 which makes quite difficult to detect the presence of defectiveness, both when represented by darker and paler areas.

\subsubsection{Bread}

330

This data set comes from industrial production of bun bread, where a digital scanner is already used to automatically assess defects concerning mainly bun dimensions, while surface defectiveness, such as dark spots, blisters, and pale areas is still evaluated by visual inspection of expert personnel. These defects arise from different causes, some of which not perfectly known, and are also often difficult to be detected by RGB online cameras. Thus, a feasibility study has been undertaken ${ }^{29}$ by acquiring offline multispectral images, covering the UV-visible range (from 430 to $700 \mathrm{~nm}, 10$ channels) and the short-wavelength NIR range (from 850 to $970 \mathrm{~nm}, 8$ channels), which can improve the acquisition of information on bread quality, combining spectral (NIR may represent also a "chemical signature") and textural information. The whole data set has 
been analysed by WT-MIA (DWT scheme) approach and described in detail in ref. 33 while here a subset of images has been analysed in order to discuss comparatively the performance of WT-MIA (DWT and SWT) and ct-MIA .

343 The raw images were cropped to remove the distortion effect of the round bun shape,

344 and background and noise were removed via preliminary wavelet analysis ${ }^{29}$, finally 345 giving images of dimensions of about 387 x 420 pixels for 18 channels. Here two nondefective images (N01, used as reference, and N02) and two defective ones (D04 and D07) are analysed, shown in Figure 6.

\section{RESULTS AND DISCUSSION}

\subsection{Artificial Images datasets}

All the three images (SimSetA, SimSetB and SimSetC) have been treated according to the same Feature Enhancement step, by considering:

- Spatial Shifting, colour-textural MIA (ct-MIA) with window size parameter $w=$ 1

- Wavelet Decomposition (WT-MIA) by using a Daubechies 1 (db1) at decomposition level $L=1$, both DWT and SWT.

357 The Feature Enhancement step gave a Feature Matrix of dimensions $I=900$ rows

358 (reduction from $32 \times 32$ to $30 \times 30$ is necessary to cope with borders) and 9 columns for the ct-MIA approach and $I=1024$ rows $\mathrm{x} 4$ columns for the WT-MIA approaches.

360 SimSetA was used as the reference set, upon which for the ct-MIA approach a Principal

361 Component Analysis model was obtained after mean centring of the Feature Matrix. 
362 Figure 7 reports the PCA results from left to right in the order scores image SimSetA, loadings, projected scores images SimSetB and SimSetC (in the order PC1 to PC4 from top to bottom). PC1 captures, for the reference ("normal") image both the difference in grey intensity value (colour) and the variation in pattern (texture) when passing from the pixels having zero value to pixels with value one, i.e. it shows the change of value when moving along the borders from one square to another, where pixel values invert, leading to a "blurring" effect of the borders. This is the expected effect since ct-MIA window of one (which is actually $3 \times 3$ pixels) moves pixel by pixel on the image structure, which is made of 16 squares of dimensions $8 \times 8$. All features contribute similarly to the PC1 loadings since the pattern change takes place in all directions. Thus, PC1 works as an average grey scale image, which in fact extracts out the spectral information (we have no other source of spectral info that a single gray scale channel). The following PCs capture only the borders effects, i.e. only the frame around the squares are visible in the scores images, and by inspecting the loadings it is possible to understand the directions of the pattern variation, e.g. to PC4 the features accounting for diagonal shift do not contribute.

When the Feature Matrix corresponding to SimSetB and SimSetC are projected onto this model, the same chequered pattern is correctly reproduced (Figure 7), but the changes in pixel correlations due to the small scale modifications of its regularity produce a large blurred area, which roughly encompasses the whole shape of the differences but extends further with respect to the faulty pixels, i.e. an area of about $3 \times 3$ pixels around each defective pixel as it is detailed in the following text. This is due to the fact that the perturbation, although being well defined (in particular for SimSetC) to 
a small number of pixels, influences the neighbouring correlation structure of all the pixels, which are contained by the moving window.

387 In a monitoring context the defective images with respect to the reference one/s can be 388 identified by the Hotelling- $\mathrm{T}^{2}$ and squared residuals (RSS, SPE or Q) multivariate control charts by using the percentage of pixels beyond control limits ${ }^{16}$. However, in this case being the images binary simply the pixel by pixel difference of the residuals sum of squares (RSS) of the test images with respect to the reference image (NOC) can be used. Both the RSS from a one or a four components PCA model are suitable to depict the faulty pixels for SimSetB and SimSetC, but also an area of about $3 \times 3$ around each faulty pixel will show up differing in RSS values with respect to NOC (Figure S2, supplementary material). This can be expected on the basis of the considerations made above on the neighbouring pixels correlation structure.

397 In the WT-MIA both decomposition scheme DWT and SWT have been applied, considering the simulated pattern, i.e. inversion of the binary value of some not consecutive pixels, db1 seems an appropriate filter. The feature matrix, holding the four decomposition blocks $\mathrm{CA}, \mathrm{CH}, \mathrm{CV}$ and $\mathrm{CD}$ (reconstructed only in DWT case), already captures the texture pattern, as highlighted in Figure 8 (DWT) and Figure 9 (SWT) 402 where the sub-images corresponding to each block of the DWT and SWT 403 decomposition of SimSetA and SimSetB are reported. This is a first difference with 404 respect to ct-MIA approach where the feature matrix holds just the shifted version of the raw image in all possible neighbouring direction (as shown in Figure S1, supplementary material) and thus PCA (in general a multivariate decomposition technique) is needed to

407 reveal the texture pattern. Further, in this case with only one reference image and one 
channel the feature analysis step by PCA is not needed at all (the decomposition blocks are orthogonal).

410 In the DWT the Approximation Block is the only one which carries the structural 411 information of image SimSetA (Figure 8, top). This is explainable by considering that 412 the db1 filter is of length two, thus operates like a window of pixel size $2 \times 2$ which 413 moves at steps of one, and due to the down-sampling scheme of DWT only one coefficient every two is retained. Thus, since the binary values change every $8 \times 8$ pixels, there is no blurring effect at the squares edges; also the coefficients in all the 416 other decomposition blocks are zeros (Figure 8, top). On the contrary, the presence of 417 deviations in the two test images, related to "sharper" structures i.e. alternating by one 418 pixel, is well captured by all decomposition blocks (Figure 8, middle). In particular Approximation (CA) shows both intensity change and texture, while Detail blocks capture horizontal $(\mathrm{CH})$, vertical $(\mathrm{CV})$ and diagonal $(\mathrm{CD})$ neighbouring pixels 421 alternation of binary values. Thinking of a monitoring context, in this case the defects 422 can be depicted by the difference between the decomposition sub-images of the 423 reference and test image, as shown in Figure 8, bottom; to this aim, considering the specific pattern of the defects in SimSetB CA and CD are the most suitable blocks. Figure 9 shows the results of the SWT decomposition. Similar considerations can be 426 drawn. The only difference is that now the effect of the variation in the binary values of 427 the pixels at the edges of the $8 \times 8$ pixels squares are visible (similarly to ct-MIA). This 428 is well explainable by the fact that in SWT down-sampling of the coefficients is not operated (to be noticed that the window size and the moving step remain the same). Analogous considerations hold for SimSetC decomposition (figure not shown for sake 431 of brevity). 
432 The behaviour of a larger filter, i.e. Daubechies $2(\mathrm{db} 2)$ of length 4 , has been also

433

434

435

436

437

438

439

440

441

442

443

444

445

446

447

448

449

450

451

452

453

454 inspected by using the SWT scheme on SimSetB (Figure S3, supplementary materials).

The db2 operates as a $4 \times 4$ window moving at steps of one pixel: the result is similar to the one obtained by ct-MIA, leading to a blurring of the borders among squares and around the area (of wideness about $3 \times 3$ ) which is interested by the defect.

Finally in Figure 10 the performance of ct-MIA and WT-MIA (SWT, db1) are compared in terms of capability of detection and localization of the faulty pixels for SimSetB (Figure 10a) and SimSetC (Figure 10b), respectively. The detection is good in both approaches being all the faulty pixels correctly identified, the difference is in the blurring area, which is strictly connected to the wideness of the analysing window, i.e. 3 x 3 for ct-MIA and $2 \times 2$ for db1. This is a general known advantage of WT of being more efficient for feature enhancement because of the availability of several filter shapes and length compared to spatial shifting approach.

4.2 Real Images datasets

4.2.1 Tiles

Several wavelet filters, belonging to Daubechies (filter length from 1 to 5), Symlet (filter length from 1 to 5), Coiflet (filter length form1 to 3) and biorthogonal (1.3 and 1.5) families were tested (decomposition levels from 1 to maximum), by using an approach as described in ref. 30. For both BZdataset and BNdataset Daubechies filter length 1 (db1 or Haar) resulted among the best performing wavelet filter and we report results relative to this filter, at decomposition level $L=3$. While for the ct-MIA approach window size 1 and 2 were considered, better performance was obtained with $w$ 
$455=1$ for BZdataset and $w=2$ for BNdataset. This lead, considering the three RGB 456 channels, to an unfolded feature matrix of size $65536 \times 27(w=1)$, or $65536 \times 75(w=$ 457 2) in the ct-MIA case, and $65536 \times 36$ in the WT-MIA case.

458 In both datasets, a single reference image has been used to calibrate the PCA models 459 and build the Hotelling's $\mathrm{T}^{2}$ and Q statistics (control charts). Autoscaling pretreatment 460 gave for both datasets and approaches the best results.

461 The choice of model dimensionality, i.e. number of principal components, in this 462 context cannot be automated, i.e. assessed on the basis of a priori fixed criterion, since it is problem dependent ${ }^{40}$. General guidelines that we adopted in this work, is to inspect how spatial features of the image are accounted for in scores images, and to scree-plot to ensure the systematic variation is modelled. Further, when enough defects images are available, to preserve some for model validation, few can be used to see which are the

467 components that maximize detection capacity. It is worth noticing that minimizing the squared prediction error in cross validation, as most used in PCA modelling, is not appropriate in this context, because it is not necessarily related to the capability of fault detection which is the objective pursued in process monitoring.

471 Image BZN01 has been used as reference NOC image for BZdataset. The PCA model 472 dimensionalities were 4 PC's for both approaches ct-MIA (captured variance $77 \%$ ) and 473 WT-MIA (captured variance 44\%), which correspond to a number of components each explaining more than $1 \%$ variance (ct-MIA) and to the first minimum in the scree-plot,

475 i.e. number of components vs. eigenvalues plot, (WT-MIA), respectively. All the 476 remaining images of the dataset were projected onto the models and distances were 477 calculated. Table 1 reports the results in terms of percentage of pixels scoring above the 
478 critical limits, which were chosen on the basis of the reference image by obtaining the $47999^{\text {th }}$ percentile values of its distances distributions. Both models are able to accept as normal behaving images BZN02 and BZN03, which are actually defectless, and 481 indicate, especially in terms of $\mathrm{T}^{2}$ distance, the presence of anomalies on all of the three 482 defective tiles, BZD01, BZD02 and BZD03; albeit the results are quite similar, a higher percentage of pixels above the critical limits is detected by the WT-MIA approach. Both approaches show similar results, although the WT-MIA identification of defects appears better defined, especially for image BZD02 where more clusters of pixels are identified, which are in particular connected to the presence of darker spots on the surface of the 487 tile, especially when using SWT (Figure S4, supplementary Material). SWT monitoring results are also shown on Table 1 and are very close to DWT one.

Interpretation of the features enhancement step can be gathered by loadings analysis: ct-MIA loadings are shown in Figure 11 (left) both as bar plot (top left) and refolded 491 (bottom left) in the corresponding position of neighbours window (the central pixel is the pixel itself). As usual PC1 is gathering an (approximately) average colour effect (all loadings have the same sign). Moreover, it can be observed that colour intensity varies left to right for red and blue channels, while green is more uniform; similarly does PC1 of WT-MIA (bar plot, top right, and decomposition sub-images, bottom right), to which contribute the Approximations of all levels and channels (Approximations in fact act as an averaging tool at each decomposition level, hence extracting out the same phenomenon as ct-MIA). From the WT-MIA Approximations sub-images (Figure 11, bottom right) it is also evident the varying intensity left to right, especially for 500 decomposition levels two and three (the green channel is uniform at level 1). This effect may be due to illumination and eventually (but was not the aim here) it could be easily 
502

503

504

505

506

507

508

509

510

511

512

513

514 As reference NOC image for BNdataset, image BNN01 has been used, the PCA model dimensionalities were 2 PC's for both approaches ct-MIA (variance captured 39\%) and

522 Neither of the models does not appear particularly satisfactory, since the normal behaving images BNN02 and BNN03, which are defectless, appear to have Q distances

524 higher than 5\%. The defective tiles, BND01 and BND03 appear above limits for both 
525 models, according to $\mathrm{T}^{2}$ distance statistic. On the contrary, defective BND02 is only 526 detected by WT-MIA, $\mathrm{T}^{2}$ distance, albeit close to the limit.

527 By considering the $\mathrm{T}^{2}$ distance values reshaped in the original pixel domain it is 528 possible to identify the groups of pixels which correspond to the passing of the critical 529 values. Figure 12a) and 12b) shows the comparison of defective images and the normal 530 images, with the corresponding distance images for ct-MIA and WT-MIA (DWT). The 531 WT-MIA identification of defects appears better defined, while ct-MIA seems to find 532 less clusters of pixels and more darker, well separated, spots all over the surface.

533 In a monitoring context, the results of Table 2 would indicate products BNN02 and 534 BNN03 as defective (false negatives) and shed doubt on rejecting or not product 535 BND02. On the other hand the possibility to look at above limits $\mathrm{T}^{2}$ distance images (or 536 in general to the images corresponding to the above limit statistic) may clarify if defects 537 are present or not. In particular, this is a case were the defects are mainly due to a non uniform distribution of pixels with a given colour content and texture that if normally 539 distributed on the image, as in the case of BNN01, BNN02 and BNN03, would be acceptable. In this situation, it may be useful to calculate and represent the local

541 entropy $^{41}$ of the scores images, were the defective area are region of low entropy 542 encircled by high entropy values, as shown in Figure 13.

543 WT-MIA model based on SWT in this case yielded lower performance.

546 The Daubechies $2(\mathrm{db} 2)$ wavelet filter was used up to decomposition level 5 and both 547 DWT and SWT decomposition schemes. The feature data matrix results of dimension 
548 Ipixels X 360 (4 blocks x 5 levels x 18 channels) is obtained. In ct-MIA both a window 549 size of 1 (162 features) and 2 (450 features) were tested. Since results were similar we 550 will discuss the ones corresponding to $w=1$, which gave a better defects localization.

551 The reference PCA model for non-defective image has been calculated by considering 552 as feature matrix the one obtained for N01 image (Figure 6). The PCA model refers to 553 mean centred data and model dimensionalities were 6 PC's for both approaches ct-MIA 554 (variance captured 66\%) and WT-MIA (variance captured 52\%), which correspond to 555 reaching the plateau in the scree-plot. We tested also a model made on two NOC images 556 but the results were analogous. Then, $\mathrm{Q}$ and $\mathrm{T}^{2}$ statistics were computed, and the critical 557 limits for each of the two statistics were computed on the basis of the 99th percentile.

558 The total percentage of pixels exceeding the critical limits is reported in Table 3. For all 559 approaches a clear detection of the two defective images can be obtained, with relevant 560 percentages of pixels above the critical limits for both distances, as well as N02 being 561 defectless.

562 However, when the $\mathrm{Q}$ and $\mathrm{T}^{2}$ values above the reference limits are refolded to the 563 original pixel $\mathrm{x}$ pixel domain to locate the defective areas on the image (Figure 14), 564 differences among the approaches emerge. ct-MIA is less efficient to detect the 565 defective area for D07 and for D04, it is also worth noticing that ct-MIA provides these 566 results when applied on the pretreated images, i.e. after denoising and background 567 removal with WT; otherwise it detects as faulty only pixels on the borders of the image. 568 DWT seems more efficient than SWT to locate the faulty pixels, notwithstanding the 569 same wavelet filter and resolution has been used.

570 Now focusing on the WT-MIA DWT results, it is worth noticing that both stains, which 571 are also easy to detect visually, but as well blisters and tiny scratches could be detected. 
572 Moreover, we can assess which features are responsible of the defects by inspecting the

$573 \mathrm{~T}^{2}$-contributions, which can be interpreted in terms of the spectral channels. In

574 particular, Figure 15 shows the $\mathrm{T}^{2}$-contributions for some of the blisters. To make the

575 representation clearer distinct plots are made for each decomposition block, and each

576 decomposition level is represented as a distinct line, so that the $\mathrm{x}$-axis reports just the

577 channels (wavelengths): the main contributions are from approximations decomposition

578 levels $1-3$, interestingly besides the visible channels some of the NIR ones $\left(11^{\text {th }}\right.$ to $18^{\text {th }}$

579 corresponding to the range from 850 to $970 \mathrm{~nm}$ at $20 \mathrm{~nm}$ resolution) contribute, which

580 point to carbohydrate, fat and water bands. This may indicate a segregation of some of

581 the ingredients on surface spots where blisters appear.

582

\section{CONCLUSIONS}

584 The artificial image datasets allowed highlighting the distinct way in which textural

585 information can be recovered by the ct-MIA and WT-MIA approaches, both are efficient

586 in depicting the salient pattern of the images and the area were the defects are located.

587 The main distinctive characteristics of the two methods are:

588 i) the feature matrix obtained by ct-MIA holds just the shifted version of the raw image

589 thus always requires coupling to a multivariate decomposition technique to highlight

590 textural patterns while the feature matrix obtained by WT-MIA already capture it;

591 ii) in general WT-MIA is more efficient for feature enhancement because of the

592 availability of several filter shapes and length compared to the spatial shifting approach

593 where only the window size can be varied. 
594 The analysis of the tiles data sets reveals a similar behaviour of the two considered 595 approaches although identification of defects appears better defined with the WT-MIA 596 approach. Also both decomposition schemes DWT and SWT show similar performance.

597 In a monitoring context it is worth noticing that when the defects are due to a non 598 uniform distribution of pixels, whose colour content and texture if normally distributed 599 on the image would be instead acceptable, further image analysis tools (e.g. local entropy or any other to assess homogeneity or heterogeneity of pixels distribution), on

601 the beyond Q or $\mathrm{T}^{2}$ limits images, are required to avoid false negative to be detected.

602 In the analysis of multispectral images (bread data set) the WT-MIA approach 603 performed better and it was possible to highlight the full benefit of the proposed 604 approach from both points of view of correct defects identification/location and 605 interpretation in terms of spectral features.

606 A further remark is that the proposed WT-MIA approach is rather straightforward 607 requiring only the Feature Extraction (Enhancement) and Reduction (Analysis) steps, as 608 in ct-MIA; one or more NOC images can be analysed at the same time and assembled in 609 the same WT features matrix which is organized pixels wise, thus allowing defect 610 localization directly. Images denoising and background removal can be as well accomplished at WT decomposition stage.

612 The proposed WT-MIA approach can be as well applied to hyperspectral images, the 613 bread data set is an example limited to eighteen channels. However, the computational 614 costs will be a limiting factor and further strategies could be considered to render it 615 more efficient, work is in progress in this direction. 
1. Esbensen K.H., Geladi P., Strategy of Multivariate Image Analysis (MIA), Chemom. Intell. Lab. Syst. 1989; 7: 67-86. comparisons, Chemom. Intell. Lab. Syst. 2004; 72: 57-71.

3. Bo S., Ding L., Li H., Di F., Zhu C., Mean shift-based clustering analysis of multispectral remote sensing imagery, International Journal of Remote Sensing 2009; 30: 817-827.

4. Villa A., Benediktsson J. A., Chanussot J., Jutten C., Hyperspectral Image Classification With Independent Component Discriminant Analysis, IEEE Transactions on Geoscience and Remote Sensing, 2011; 49: 4865-4876.

5. JohnN. M., Kabuka M. R., Ibrahim M. O., Multivariate statistical model for 3D image segmentation with application to medical images, Journal of Digital Imaging, 2003; 16: 365-377.

6. Nattkemper T. W., Multivariate image analysis in biomedicine, Journal of Biomedical Informatics, 2004; 37: 380-391.

7. Hackmack K., Paul F., Weygandt M., Allefeld C., Haynes J. D., Multi-scale classification of disease using structural MRI and wavelet transform, NeuroImage 2012; 62: 48-58.

8. Sanz-Requena, R; Prats-Montalbán, J.M.; Martí-Bonmatí, L.; Ferrer, A.; Alberich-Bayarri, A.; García-Martí, G.; Pérez, R. Automatic Individual Arterial Input Functions Calculated From PCA Outperform Manual and Population-Averaged Approaches for the Pharmacokinetic Modeling of DCE-MR Images. Journal of Magnetic Resonance Imaging, 2015; 42: 477-487.

9. Prats-Montalbán J.M., Aguado E.,Ferrer A.. Chapter 16: Multivariate Curve Resolution for Magnetic Resonance Image analysis: applications in prostate cancer biomarkers development. In Ruckebusch $\mathrm{C}$ ed. "Resolving Spectral Mixtures, with application from ultrafast spectroscopy to super-resolution imaging", Data Handling in Science and Technology 30, Elsevier, 2017: 519-550.

10. Liu J., MacGregor J., On the extraction of spectral and spatial information from images, Chemom. Intell. Lab. Syst. 2007; 85: 119-130.

11. Duchesne C., Liu J. J., MacGregor J. F., Multivariate image analysis in the process industries: A review, Chemom. Intell. Lab. Syst. 2012; 117: 116-128.

12. Prats-Montalban J. M., de Juan A., Ferrer A., Multivariate image analysis: A review with applications, Chemom. Intell. Lab. Syst. 2011; 107: -23.

13. Reis M.S., Multivariate image analysis. In: Granato D., Ares G. ed. Mathematical and Statistical Methods in Food Science and Technology. Chichester: Wiley-Blackwell; 2014: 201-218. ISBN: 978-1-118-43368-3.

14. Facco P., Masiero A., Beghi A., Advances on multivariate image analysis for product quality monitoring, Journal of Process Control, 2013; 23: 89-98.

15. Antonelli A., Cocchi M., Fava P., et al., Automated evaluation of food colour by means of multivariate image analysis coupled to a wavelet-based classification algorithm, Anal. Chim. Acta, 2004; 515: 3-13.

16. Prats-Montalban J.M., Ferrer A., Statistical process control based on Multivariate Image Analysis: A new proposal for monitoring and defect detection, Computers and Chemical Engineering 2014; 74: 501-511.

17. Prats-Montalban J.M., Ferrer A., Integration of colour and textural information in multivariate image analysis: defect detection and classification issues, J. Chemom. 2007; 21: $10-23$. 
18. Reis M.S., An integrated multiscale and multivariate image analysis framework for process monitoring of colour random textures: MSMIA. Chemom. Intell. Lab. Syst. 2015; 142: 3648.

19. Kourti T., Process Analytical Technology Beyond Real-Time Analyzers: The Role of Multivariate Analysis, Critical Reviews in Analytical Chemistry, 2006; 36: 257-278.

20. Bharati M., MacGregor J. F., Softwood lumber grading through on line multivariate image analysis, Industrial and Engineering Chemistry Research, 2003; 42: 5345-5353.

21. Boldrini B., Kessler W., Rebner K., Kessler R. W., Hyperspectral imaging: a review of best practice, performance and pitfalls for in-line and on-line applications J. Near Infrared Spectroscopy, 2012; 20: 483-508.

22. Liu J. J., MacGregor J. F., Estimation and monitoring of product aesthetics: application to manufacturing of "engineered stone" countertops Machine Vision and Applications, 2006; 16: 374-383.

23. Elmasry G., Kamruzzaman M., Sun D. W., Allen P., Principles and Applications of Hyperspectral Imaging in Quality Evaluation of Agro-Food Products: A Review, Critical Reviews in Food Science and Nutrition, 2012; 52: 999-1023.

24. Ganesan R., Das T. K., Venkataraman V., Wavelet-based multiscale statistical process monitoring: A literature review, IIE Transactions, 2004; 36: 787-806.

25. Juneau P., Garnier A., Duchesne C., The undecimated wavelet transform - multivariate image analysis (UWT-MIA) for simultaneous extraction of spectral and spatial information, Chemom. Intell. Lab. Syst., 2015; 142: 304-318.

26. Reis M. S., Bauer A., Wavelet texture analysis of on-line acquired images for paper formation assessment and monitoring, Chemom. Intell. Lab. Syst., 2009; 95: 129-137.

27. Facco P., Tomba E., Roso M., et al., Automatic characterization of nanofiber assemblies by image texture analysis, Chemome. Intell. Lab. Syst. 2010; 103: 66-75.

28. Facco P., Santomaso A.C., Barolo M., Artificial vision system for particle size characterization from bulk materials, Chemical Engineering Science 2017; 164: 246-257.

29. Li Vigni M., Cocchi M., Chpt. 13 Multiresolution Analysis and Chemometrics for Pattern Enhancement and Resolution in Spectral Signals and Images, In Ruckebusch C ed. "Resolving Spectral Mixtures, with application from ultrafast spectroscopy to superresolution imaging", Data Handling in Science and Technology 30, Elsevier, 2017 :

30. Pesquet J.C., Krim H., Carfantan H. Time invariant orthonormal wavelet representation. IEEE Transactions on Signal Processing, 1996; 44: 1964-1970.

31. Coifman R., Donoho D. Translation-invariant de-noising. In Wavelets and Statistics, (ed). Springer Verlag, Lecture Notes in Statistics: New York, 1995, 125-130.

32. Mallat S. A theory for multi-resolution signal decomposition: the wavelet representation. IEEE Trans. Pattern Anal. Mach. Intell. 1989; 11: 674-693.

33. Cohen A., Daubechies I., Jawerth B., Vial P., Multiresolution analysis, wavelets and fast wavelet transform on an interval. CRAS Paris, Ser. A, 1993; 316: 417-421.

34. Mojsilovic A., Popovic V., Rackov D. M., On the selection for an optimal wavelet basis for texture characterization. IEEE Trans. Image Process. 2000; 9: 2043-2050.

35. Villasenor J. D., Belzer B., Liao J., Wavelet filter evaluation for image compression. IEEE Trans. Image Process., 1995; 4: 1053-1060.

36. Svensson O., Abrahamsson K., Engelbrektsson J., et al., An evaluation of 2D-wavelet filters for estimation of differences in textures of pharmaceutical tablets, Chemom. Intell. Lab. Syst., 2006; 84: 3-8.

37. Prats-Montalban J. M., Cocchi M., Ferrer A., N-way modeling for wavelet filter determination in multivariate image analysis. J Chemometr. 2015; 29: 379-88.

38. Mallet Y., de Vel O., Coomans D., Fundamentals of wavelet transforms. In Wavelets in 
Chemistry, (ed). Elsevier Science B. V.: Amsterdam, 2000: 77-84.

714 39. Misiti M., Wavelet Toolbox User’s Guide, MathWorks: Massachusetts, 1996.

715 40. Camacho J., Ferrer A., Cross-validation in PCA models with the element wise k-fold (ekf) algorithm: practical aspects. Chemom Intell Lab Syst, 2014; 131: 37-50.

719 Jersey, Prentice Hall, 2003. 
720 Table 1. BZDataset. Percentage of pixels above Hotelling's $\mathrm{T}^{2}$ and Residuals Q 721 distances critical limits based on normal image BZN01 $99^{\text {th }}$ percentile

\begin{tabular}{|c|c|c|c|c|c|c|}
\hline & \multicolumn{2}{|c|}{$\begin{array}{c}\text { ct-MIA } \\
w=1,4 \text { PCs }\end{array}$} & \multicolumn{2}{|c|}{$\begin{array}{c}\text { WT-MIA (DWT) } \\
\text { Daubechies } 1 \text {, level = } 3 \\
4 \text { PCs }\end{array}$} & \multicolumn{2}{|c|}{$\begin{array}{c}\text { WT-MIA }(\mathbf{S W T}) \\
\text { Daubechies } 1, \text { level = } 3 \\
4 \text { PCs }\end{array}$} \\
\hline & $T^{2}$ distance & $Q$ distance & $T^{2}$ distance & $Q$ distance & $\mathrm{T}^{2}$ distance & Q distance \\
\hline BZN01 & $1.0 \%$ & $1.0 \%$ & $1.0 \%$ & $1.0 \%$ & $1.0 \%$ & $1.0 \%$ \\
\hline BZN02 & $0.6 \%$ & $0.6 \%$ & $0.6 \%$ & $0.7 \%$ & $0.7 \%$ & $0.6 \%$ \\
\hline BZN03 & $0.9 \%$ & $0.9 \%$ & $0.8 \%$ & $0.7 \%$ & $0.8 \%$ & $0.7 \%$ \\
\hline BZD01 & $3.6 \%$ & $1.9 \%$ & $5.1 \%$ & $2.8 \%$ & $4.1 \%$ & $3.0 \%$ \\
\hline BZD02 & $1.6 \%$ & $0.7 \%$ & $2.5 \%$ & $0.7 \%$ & $1.9 \%$ & $0.9 \%$ \\
\hline BZD03 & $1.9 \%$ & $0.9 \%$ & $2.4 \%$ & $0.7 \%$ & $2.1 \%$ & $0.8 \%$ \\
\hline
\end{tabular}

722 
724 Table 2. BNDataset. Percentage of pixels above Hotelling's $\mathrm{T}^{2}$ and Residuals Q distances critical limits based on normal image BNN01 95 ${ }^{\text {th }}$ percentile

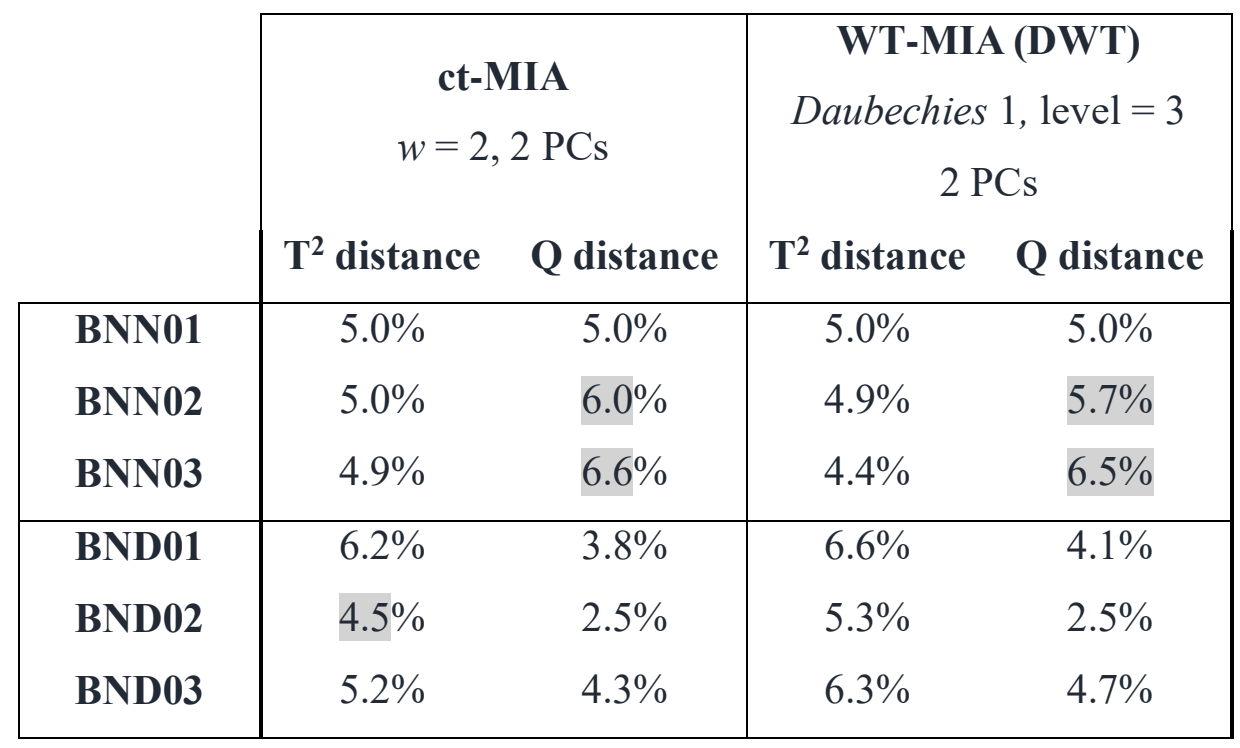

726

727

728

729 
730 Table 3. Bread Dataset. Percentage of pixels above Hotelling's $\mathrm{T}^{2}$ and Residuals Q

731 distances critical limits based on normal image N01 $99^{\text {th }}$ percentile

\begin{tabular}{|c|cc|cc|cc|}
\hline & \multicolumn{2}{|c|}{ ct-MIA } & \multicolumn{2}{c|}{$\begin{array}{c}\text { WT-MIA (DWT) } \\
\text { Daubechies 2, level = 5 }\end{array}$} & \multicolumn{2}{c|}{$\begin{array}{c}\text { WT-MIA (SWT) } \\
\text { Daubechies 2, level = 5 }\end{array}$} \\
& \multicolumn{2}{|c|}{$w=1,6 \mathrm{PCs}$} & \multicolumn{2}{c|}{ 6 PCs } & \multicolumn{2}{c|}{6 PCs } \\
N01 & $1.0 \%$ & $1.0 \%$ & $1.0 \%$ & $1.0 \%$ & $1.0 \%$ & $1.0 \%$ \\
N02 & $0.6 \%$ & $1.0 \%$ & $0.5 \%$ & $0.7 \%$ & $0.9 \%$ & $0.2 \%$ \\
\hline D04 & $2.7 \%$ & $3.4 \%$ & $3.3 \%$ & $3.2 \%$ & $11.9 \%$ & $11.4 \%$ \\
D07 & $4.3 \%$ & $3.0 \%$ & $4.6 \%$ & $4.3 \%$ & $15.7 \%$ & $14.3 \%$ \\
\hline
\end{tabular}

732

733

734

735 